\title{
Ballistic lobar trajectory outcomes in civilian firearm penetrating brain injury
}

\author{
Georgios Alexopoulos, MD, ${ }^{1,2}$ Nabiha Quadri, MD, 1,2, Maheen Khan, MD, ${ }^{1,2}$ Henna Bazai, BS, ${ }^{2}$ \\ Carla Formoso Pico, BS, ${ }^{2}$ Connor Fraser, BS, ${ }^{2}$ Neha Kulkarni, BS, ${ }^{2}$ Joanna Kemp, MD, ${ }^{1,2}$ \\ Jeroen Coppens, MD, ${ }^{1,2}$ Richard Bucholz, MD, ${ }^{1,2}$ and Philippe Mercier, MD, PhD ${ }^{1,2}$
}

${ }^{1}$ Department of Neurosurgery and ${ }^{2}$ School of Medicine, Saint Louis University, St. Louis, Missouri

OBJECTIVE Penetrating brain injury (PBI) is the most lethal of all firearm injuries, with reported survival rates of less than $20 \%$. The projectile trajectory (PT) has been shown to impact mortality, but the significant lobar tracks have not been defined. The aim of this retrospective case-control study was to test for associations between distinct ballistic trajectories, missile types, and patient outcomes.

METHODS A total of 243 patients who presented with a PBI to the Saint Louis University emergency department from 2008 through 2019 were identified from the hospital registry. Conventional CT scans combined with 3D CT reconstructions and medical records were reviewed for each patient to identify distinct PTs.

RESULTS A total of 65 ballistic lobar trajectories were identified. Multivariable regression models were used, and the results were compared with those in the literature. Penetrating and perforating types of PBI associated with bitemporal (t-statistic $=-2.283, p=0.023$ ) or frontal-to-contralateral parietal (t-statistic $=-2.311, p=0.025$ ) projectile paths were universally found to be fatal. In the group in which the Glasgow Coma Scale (GCS) score at presentation was lower than 8 , a favorable penetrating missile trajectory was one that involved a single frontal lobe (adjusted OR 0.02 [ $95 \% \mathrm{Cl} 0.00-$ 0.38 ], $p=0.022$ ) or parietal lobe (adjusted OR 0.15 [95\% Cl 0.02-0.97], $p=0.048$ ). Expanding or fragmenting types of projectiles carry higher mortality rates (OR 2.53 [95\% Cl 1.32-4.83], $p<0.001)$ than do nondeformable missiles. Patient age was not associated with worse outcomes when controlled by other significant predictive factors.

CONCLUSIONS Patients with penetrating or perforating types of PBI associated with bitemporal or frontal-to-contralateral parietal PTs should be considered as potential donor candidates. Trauma patients with penetrating missile trajectories involving a single frontal or parietal lobe should be considered for early neurosurgical intervention, especially in the circumstances of a low GCS score $(<8)$. Surgeons should not base their decision-making solely on advanced patient age to defer further treatment. Patients with PBls caused by nondeformable types of projectiles can survive multiple simultaneous intracranial missile trajectories.

https://thejns.org/doi/abs/10.3171/2020.6.JNS201837

KEYWORDS penetrating brain injury; ballistic trajectory; penetrating brain trauma; gunshot wound to the head; ballistic outcomes; traumatic brain injury

$\mathrm{P}$ ENETRATING brain injury (PBI) related to firearms has been associated with high mortality and uncertain recovery. ${ }^{1-11}$ The United States leads in PBI mortality among all high-income nations, with more than 32,000 people dying and more than 67,000 people injured each year. ${ }^{1-3,6,8-10}$ Between 2006 and 2010, in the United States, PBI resulted in an estimated 385,769 emergency visits and 141,914 hospital admissions, costing $\$ 88.6$ billion..$^{1,9,10}$

A Centers for Disease Control and Prevention study found that PBI homicide rates were higher in metropolitan areas in the Midwest (7 of 10 above the median rate of 5.4 per 100,000). ${ }^{12}$ Saint Louis University Hospital is a level 1 adult trauma center serving the region that has led local efforts to promote awareness about this significant problem.

The lack of clear guidelines on PBI management calls for further understanding of PBI outcomes and predictors of mortality. ${ }^{1,2}$ Determining which patients will require aggressive treatment is necessary, but it is still unclear which subjects will benefit from such management. ${ }^{11,12}$ The projectile trajectory (PT) has been shown to impact both mortality and morbidity after PBI, but the individual significant lobar trajectories have not been defined., ${ }^{4,14,15}$

ABBREVIATIONS EVD = external ventricular drain; GCS = Glasgow Coma Scale; GOS = Glasgow Outcome Scale; IVH = intraventricular hemorrhage; PBI = penetrating brain injury; $\mathrm{PT}=$ projectile trajectory.

SUBMITTED May 15, 2020. ACCEPTED June 16, 2020.

INCLUDE WHEN CITING Published online November 6, 2020; DOI: 10.3171/2020.6.JNS201837. 
The shape, type, and location of the PT and retained missile fragments also correlate with clinical outcomes. CT scanning has emerged as the most important tool in the analysis of ballistic paths, especially when combined with $3 \mathrm{D}$ reconstruction. ${ }^{6,16,17}$ To our knowledge, there have been no studies to test the relationships between CT-based projectile path localization, gunshot wound type, and clinical outcomes.

We attempted to test the hypothesis that clinical outcomes after PBI are independent of the underlying ballistic trajectory and/or missile type.

\section{Methods}

\section{Patients and Data Collection}

We identified 245 patients with PBI who presented to Saint Louis University Hospital during a 12-year period extending from January 2008 to December 2019. Only patients with CT scans available for review were included in the study. Demographics, clinical information, presence of associated injuries, and outcomes were collected for each patient. The medical records were reviewed for surgical procedures, length of hospital stay, death attributed to other causes, and organ donation (Table 1). The Glasgow Outcome Scale (GOS) was used as a measure of patient survival, with the primary outcome being GOS score at the time of discharge; for patients who survived their injuries, we used the GOS score at a follow-up 3 months after the injury. Two individuals were excluded from the analysis, as their deaths were attributed to other causes; the first patient died of severe thoracoabdominal injuries, while the death of the second patient was due to a massive pulmonary embolism. A second analysis of the entire sample followed in which we included all 245 patients, and, by comparing the two analyses, we evaluated for alterations in the effect sizes among groups that could have been attributed to the two excluded cases. This study was approved by the ethics review board of Saint Louis University Hospital.

\section{Methods}

The CT scans of 243 patients were reviewed retrospectively by two attending neurosurgeons from our department who remained blinded to the results and demographic data. Three-dimensional CT reconstructions were obtained for each patient using the volume-rendering technique for identification of the bullet entry and exit wounds and intracranial trajectory (Fig. 1), as described by Tartaglione et al. ${ }^{16,18,19}$ Known anatomical landmarks were used to describe the projectile entry and exit sites. With the integration of a 3D stereotactic atlas of the human skull, we recognized five possible options as points of entry or exit for projectiles (frontal, parietal, temporal, occipital, and suboccipital). ${ }^{20,21}$ We could identify four major gunshot wound types: penetrating (missile entry through the skull without exit wound, and bullet lodged intracranially), perforating (through and through intracranial PTs with both entry and exit wounds), tangential (projectile injuries that do not penetrate through the skull), and careening (projectile penetrates the skull and travels along the periphery of the bone cortex without intracranial en- try). ${ }^{6,7}$ The surgeons assessed each CT scan to identify a discrete PT defined by the anatomical entry and possible exit sites, as well as the specific type of gunshot wound (perforating, penetrating, careening, tangential) for every patient, $, 6,6,17,22,23$ the exact projectile type (expanding or fragmenting vs nondeformable bullets) based on the presence or absence of a trail of lead fragments (Fig. 2), ${ }^{23-25}$ the location of intracranial fragments (bihemispheric vs unihemispheric), and the space-occupying lesion as follows: extraaxial hematoma $\geq 10 \mathrm{~mm}$ in thickness and/or intraparenchymal hemorrhage $>30 \mathrm{ml}$ in volume. The intracerebral hemorrhage volumes were calculated for each patient using the $\mathrm{ABC} / 2$ formula. ${ }^{26}$

The estimations of two neurosurgeons were then compared for potential discrepancies. The first surgeon (J.K.) identified 65 intracranial PTs, while the second surgeon (J.C.) recognized 68 discrete PTs. The point of disagreement was the bullet entry side in all 3 cases; 2 cases were estimated as a midline entry (J.K.) in contrast to a leftside entry (J.C.), and 1 case was estimated as right-side entry (J.K.) in contrast to a midline side (J.C.). There were no disagreements among the surgeons regarding the bullet entry site, exit site, anatomical lobar compartment involvement, or projectile type. The gunshot wound type was also a matter of debate, since the first surgeon (J.K.) classified 4 cases as careening while the second surgeon (J.C.) classified the same cases as tangential. To eliminate the selection bias, a third neurosurgeon (R.B.) was called to review the CT scans of all 7 cases where a disagreement arose. In case a disagreement remained between the three reviewers, our plan was to exclude that specific patient from analysis. With the aforementioned methods, PTs and missile types were easy to identify in all patients, so no subject identified with a PBI in the registry was excluded from our sample. Combining CT analysis and 3D volumetric reconstruction allowed for the identification of 65 discrete intracranial PTs.

\section{Statistical Analysis}

Descriptive analysis for each individual variable was conducted. Median, IQR, and mean were calculated for numeric variables, and differences were compared using the Wilcoxon rank-sum test. Categorical parameters are presented as numbers, with groups compared using the chi-square or Fisher's exact test, as appropriate. Bivariate analysis continued by calculating the odds ratios and their 95\% confidence intervals to test the strength of association for each categorical variable. Multivariable analysis was performed using regression models to calculate the t-statistic for each explanatory variable and test for a regression relationship with the dependent variable, or GOS score. The best predicting variables to fit our model were selected by calculating the partial f-tests. The regression model was controlled for multiple confounders. We stratified the sample into groups to control confounding and then calculated the adjusted OR. The confounding variables were selected according their significance in the bivariate analysis as well as the coefficient of multiple determination $\left(\mathrm{R}^{2}\right)$ values in the regression model; $\mathrm{p} \leq 0.05$ was considered significant. RStudio version 1.2.5042 software was used for the statistical analysis. 
TABLE 1. Univariate and bivariate analyses: testing for association between patient demographics, clinical variables, radiographic characteristics, ballistics, and patient mortality $(n=243)$

\begin{tabular}{|c|c|c|c|}
\hline Parameter & Value & Wilcoxon or OR $(95 \% \mathrm{Cl})^{*}$ & $\mathrm{p}$ Value \\
\hline Age, yrs & & Wilcoxon 4.01 (1.01-7.99) & 0.003 \\
\hline Mean & 32.6 & & \\
\hline Median (IQR) & $28(21.5-40)$ & & \\
\hline Sex & & & 0.475 \\
\hline Male & $205(84.4)$ & & \\
\hline Female & $38(15.6)$ & & \\
\hline LOC & & & - \\
\hline Yes & $194(79.8)$ & & \\
\hline No & $49(20.2)$ & & \\
\hline Presence of skull fracture & & & - \\
\hline Yes & $221(90.9)$ & & \\
\hline No & $22(9.1)$ & & \\
\hline Intracranial bullet fragments & & & $<0.001$ \\
\hline Yes & $179(73.7)$ & OR 16.84 (7.45-38.05) & \\
\hline No & $64(26.3)$ & & \\
\hline Bullet entry side & & & 0.932 \\
\hline $\mathrm{Lt}$ & $111(45.7)$ & & \\
\hline $\mathrm{Rt}$ & $121(49.8)$ & & \\
\hline Midline & $9(3.7)$ & & \\
\hline Multiple & $2(0.8)$ & & \\
\hline Gunshot wound type & & & $<0.001$ \\
\hline Perforating & $42(17.3)$ & OR 35.83 (8.41-152.62) & \\
\hline Penetrating & $137(56.4)$ & OR 3.05 (1.75-5.31) & \\
\hline Careening & $32(13.2)$ & OR $0.06(0.02-0.17)$ & \\
\hline Tangential & $32(13.2)$ & OR $0.28(0.15-0.53)$ & \\
\hline Intracranial fragments' location $(n=179)$ & & & $<0.001$ \\
\hline Unihemispheric & $89(49.7)$ & OR $0.06(0.03-0.13)$ & \\
\hline Bihemispheric & $90(50.3)$ & OR $16.84(7.45-38.05)$ & \\
\hline Intracranial missile fragments (bullet type; $n=179$ ) & & & $<0.001$ \\
\hline Expanding/fragmenting & $75(41.9)$ & OR $2.53(1.32-4.83)$ & \\
\hline Nondeformable & $104(58.1)$ & OR $0.40(0.21-0.76)$ & \\
\hline Bihemispheric projectile trajectory $(n=179)$ & & & $<0.001$ \\
\hline Yes & $97(54.2)$ & OR 27.28 (10.09-73.78) & \\
\hline No & $82(45.8)$ & & \\
\hline Intraventricular hemorrhage & & & $<0.001$ \\
\hline Yes & $67(27.6)$ & OR $31.25(11.87-82.29)$ & \\
\hline No & $176(72.4)$ & & \\
\hline CT scan, midline shift, $\mathrm{mm}$ & & Wilcoxon 2.01 (1.99-2.99) & $<0.001$ \\
\hline Mean & 3.14 & & \\
\hline Median (IQR) & $2(0-4)$ & & \\
\hline Pneumocephalus & & & 0.589 \\
\hline Yes & $204(84.0)$ & & \\
\hline No & $39(16.0)$ & & \\
\hline \multicolumn{4}{|l|}{ Isolated gunshot wound to the head } \\
\hline Yes & $201(82.7)$ & & \\
\hline No & $42(17.3)$ & & \\
\hline
\end{tabular}


» CONTINUED FROM PAGE 3

TABLE 1. Univariate and bivariate analyses: testing for association between patient demographics, clinical variables, radiographic characteristics, ballistics, and patient mortality $(n=243)$

\begin{tabular}{lccc}
\hline \multicolumn{1}{c}{ Parameter } & Value & Wilcoxon or OR $(95 \% \text { Cl })^{*}$ & $p$ Value \\
\hline GCS score at presentation & & & $<0.001$ \\
\hline $3-7$ & $133(54.7)$ & OR $68.05(26.98-171.62)$ & \\
\hline $8-12$ & $39(16.0)$ & OR 0.17 (0.07-0.42) & \\
\hline $13-15$ & $71(29.2)$ & OR 0.00 (0.00-0.06) & 0.01 \\
\hline Pupils unequal $(n=207)$ & & & \\
\hline Yes & $30(14.5)$ & OR 3.03 (1.31-6.99) & $<0.001$ \\
\hline No & $177(85.5)$ & & $<0.001$ \\
\hline Pupils or pupil reactive & & & \\
\hline Yes & $147(60.5)$ & OR 0.01 (0.00-0.03) & \\
\hline No & $96(39.5)$ & & \\
\hline Space-occupying lesion† & & & \\
\hline Yes & $80(32.9)$ & OR 2.72 (1.56-4.72) & \\
\hline No & $163(67.1)$ & & \\
\hline
\end{tabular}

$\mathrm{LOC}=$ loss of consciousness following the injury.

Values are the number of patients (\%) unless stated otherwise. When a dichotomous categorical value is present, only the OR for the answer "yes" is provided in the table.

* Tests for categorical values represent the odds ratio (OR; $95 \% \mathrm{Cl})$. Tests for numeric values represent the median difference in a Wilcoxon rank-sum test $(95 \% \mathrm{Cl})$.

$\dagger$ Defined as subdural hematoma $>10 \mathrm{~mm}$ in thickness and/or intracerebral hemorrhage $>30 \mathrm{ml}$ in volume.
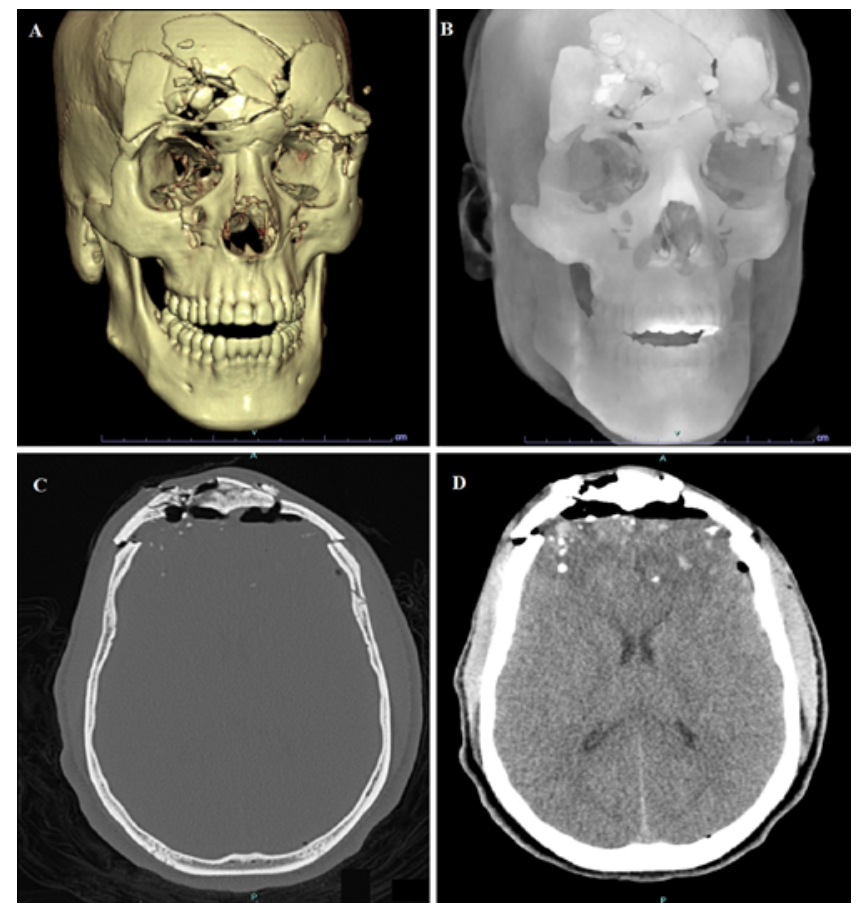

FIG. 1. Three-dimensional shaded surface rendering in a patient with a penetrating type of injury due to an expanding or fragmenting type of missile. A: The projectile fragments enter the dura through the right frontal bone, causing a severely comminuted fracture with involvement of the frontal sinuses. B: In 3D volume rendering, various parameters such as surface shading can simplify identification of the projectile entry site. C and D: Respective bone (C) and brain (D) windows on axial CT scans at the level of the injury, revealing multiple missile fragments in the ipsilateral frontal lobe and bone fragments located in bilateral frontal lobes. Figure is available in color online only.

\section{Results}

\section{Patient Demographics}

A total of 243 patients with PBI presented to our institution between 2008 and 2019. The mean age ( \pm SD) was $32.6 \pm 14.1$ years (Table 1$)$. Increased patient age was associated with adverse outcomes in the bivariate analysis; the median age difference of patients with a GOS score of 1 was 4 years older than survivors (Wilcoxon 4.01 [95\% CI
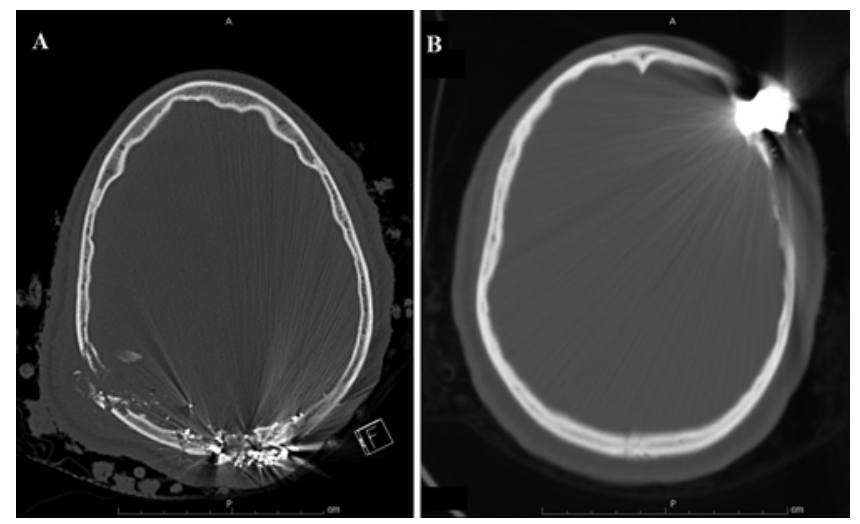

FIG. 2. Difference between expanding/fragmenting and nondeformable types of projectiles. A: Expanding and fragmenting bullets are designed to expand on impact. The violent deformation may shed fragments as they expand. These fragments act as secondary missiles and lead to more direct tissue destruction. This type of bullet can cause devastating injuries to victims; it is easily identifiable on the CT scan as it leaves a telltale trail of metal fragments. B: Nondeformable types of projectiles are the usual "military bullets." They are made of lead core surrounded by a hard metal casing. Nondeformable projectiles often remain in one piece and do not leave a trail of lead sign along their path. 
TABLE 2. Respective intracranial ballistic trajectories that favor survival and relative death odds ratio for each individual path $(n=89 / 179)$

\begin{tabular}{lccc}
\hline $\begin{array}{c}\text { Anatomic } \\
\text { Bullet Entry } \\
\text { Site }\end{array}$ & $\begin{array}{c}\text { Anatomic Bullet } \\
\text { Exit Site or Lodged } \\
\text { Intracranial } \\
\text { Compartment }^{*}\end{array}$ & $\begin{array}{c}\text { No. of } \\
\text { Patients }\end{array}$ & $\begin{array}{c}\text { Death OR } \\
(95 \% \mathrm{Cl})\end{array}$ \\
\hline Frontal & Contralateral frontal & 4 & $0.51(0.08-4.30)$ \\
\hline Frontal & Contralateral occipital & 3 & $0.20(0.04-8.49)$ \\
\hline Frontal & Frontal & 13 & $\mathbf{0 . 0 2 ( 0 . 0 0 - 0 . 3 8 )}$ \\
\hline Frontal & Parietal & 14 & $0.41(0.19-1.12)$ \\
\hline Frontal & Temporal & 5 & $0.48(0.04-6.99)$ \\
\hline Multiple & NA & 2 & $0.59(0.04-9.67)$ \\
\hline Occipital & Occipital & 5 & $0.12(0.01-2.46)$ \\
\hline Occipital & Parietal & 3 & $0.20(0.04-4.91)$ \\
\hline Parietal & Frontal & 11 & $0.32(0.09-1.13)$ \\
\hline Parietal & Parietal & 10 & $\mathbf{0 . 1 2 ( 0 . 0 2 - 0 . 5 6 )}$ \\
\hline Suboccipital & Parietal & 1 & $0.20(0.01-4.91)$ \\
\hline Suboccipital & Suboccipital & 1 & $0.20(0.01-4.91)$ \\
\hline Temporal & Frontal & 4 & $0.51(0.08-4.30)$ \\
\hline Temporal & Occipital & 2 & $0.59(0.04-9.67)$ \\
\hline Temporal & Parietal & 4 & $0.59(0.08-4.30)$ \\
\hline Temporal & Temporal & 7 & $\mathbf{0 . 0 4}(\mathbf{0 . 0 0}-0.76)$ \\
\hline
\end{tabular}

$\mathrm{NA}=$ not applicable

Statistically significant trajectories are shown in bold.

* Refers to ipsilateral lobar compartments unless stated otherwise.

1.01-7.99], $p=0.002)$. The age distribution of patients was positively skewed, with most injuries occurring in the 2nd and 3 rd decades of life. There was a male predominance of 205 (84.4\%) patients. Sex showed no association, and the difference in the odds of death between males and females was not statistically significant $(\mathrm{p}=0.475)($ Table 1$)$.

\section{Physical Examination at Presentation}

The Glasgow Coma Scale (GCS) score at presentation was classified into three groups: scores 3-7, 8-12, and 13-15. In the bivariate analysis, a GCS score of 3-7 was associated with a higher mortality rate (OR 68.05 [95\% CI 26.98-171.62], $\mathrm{p}<0.001)$. Patients with GCS scores of 8-12 had an $83 \%$ lower chance of death (OR 0.17 [95\% CI 0.07-0.42], $\mathrm{p}<0.001$ ), and patients with GCS scores of $13-15$ almost universally survived (OR 0.00 [95\% CI 0.00-0.06], p < 0.001) (Table 1).

The status of patients with at least one reactive pupil at presentation was associated with increased survival (death OR 0.01 [95\% CI 0.00-0.03], p < 0.001). Unequal pupils were associated with a poor outcome (OR 3.03 [95\% CI 1.31-6.99], $\mathrm{p}=0.01)($ Table 1).

\section{Radiographic Findings}

Intraventricular hemorrhage (IVH) was associated with poor outcome (OR 31.25 [95\% CI 11.87-82.29], $\mathrm{p}<$ 0.001 ), while pneumocephalus on the CT scan was not significantly associated with mortality. Patients with spaceoccupying lesions had 2.72 times higher odds of dying
(95\% CI 1.56-4.72, p < 0.001). The median difference of shift as measured in CT scans was approximately $2 \mathrm{~mm}$ higher in patients who had GOS scores of 1 (Wilcoxon 2.01 [95\% CI 1.99-2.99], $\mathrm{p}<0.001$ ) (Table 1).

\section{Ballistics \\ Projectile Wound Type and Missile Fragment Type}

Most (56.4\%) injuries were classified as a penetrating gunshot wound type. Penetrating and perforating types of PBIs were also associated with death, particularly with the odds of death in patients with perforating injuries, which was 35.83 times higher (95\% CI 8.41-152.62, $\mathrm{p}<0.001$ ). The odds of death in patients with careening (OR 0.06 [95\% CI 0.02-0.17], $\mathrm{p}<0.001$ ) and tangential (OR 0.28 [95\% CI 0.15-0.53], p < 0.001) wound types were 94\% and $72 \%$ lower than in the rest of the population, respectively. Expanding or fragmenting projectile types caused significantly greater damage, and patients with those injuries had 2.53 times higher odds of dying (95\% CI 1.32-4.83, p < 0.001) compared with patients with injuries from nondeformable projectiles (Table 1).

\section{Projectile Entry Side/Site and Intracranial Fragments}

The entry side (left vs right vs midline) of the projectile was not associated with adverse outcomes $(p=0.932)$. The most common entry site in the skull was frontal $(\mathrm{n}=96$, $39.5 \%)$, followed by parietal $(n=62,26 \%)$ and temporal ( $n$ $=59,24 \%)$. The presence of intracranial bullet fragments was associated with death $(\mathrm{p}<0.001)$; more specifically, the odds of patients dying of intracranial projectile fragments located in bilateral hemispheres was 16.84 times greater $(95 \%$ CI $7.45-38.05, \mathrm{p}<0.001)$ (Table 1).

\section{Significance of Ballistic Lobar Trajectory}

To further categorize the 65 intracranial PTs into groups, we removed the factor of the side of bullet entry from the equation because it did not impact the outcome, as already shown. With respect to lobar anatomy and bihemispheric PT, we grouped together all bilateral PTs with identical intracranial anatomical paths, but where the points of entry were contralateral (midline entry sites were also grouped together). By doing this, we ended up identifying 32 discrete PTs (Tables 2 and 3). Finally, we excluded from further analysis all patients with careening and tangential wounds because those types of PBI were universally associated with favorable outcomes in the bivariate analysis.

We focused on the subpopulation that sustained penetrating and perforating PBIs to identify significant PTs. This yielded a total of 179 patients. Perforating bullet paths entering the dura through the frontal bone, with the missile fragments located in the ipsilateral frontal lobe, had favorable outcomes (death OR 0.02 [95\% CI 0.00-0.38], $\mathrm{p}<0.001$ ) (Table 2). The results were similar for perforating PTs entering the dura through the parietal or temporal bones and lying in the ipsilateral parietal or temporal lobe. Tracts that were found to be universally fatal were bitemporal PTs (OR of survival 0.05 [95\% CI 0.00-0.79], p < 0.001) (Table 3) and tracts entering the skull through the frontal bone and then traversing the midline and entering 
the contralateral parietal lobes (OR of survival 0.03 [95\% CI 0.00-0.56], $\mathrm{p}<0.001$ ) (Table 3). The odds of dying for patients with a bihemispheric PT were 27.28 times higher (95\% CI 10.09-73.78, $\mathrm{p}<0.001)$ than for the remaining population (Table 1).

\section{Multivariable Regression Analysis}

We tried to identify the optimal regression model by testing the effect of multiple predictors on the explanatory power of our analysis. By integrating midline shift (partial f-test, $\mathrm{p}<0.001$ ) and GCS score at presentation (partial f-test, $p<0.001$ ) as continuous explanatory variables and GOS score as the outcome variable, we identified the best predictive model (multiple $\mathrm{R}^{2}, 0.9373$; adjusted $\mathrm{R}^{2}, 0.9117$; predicted $\mathrm{R}^{2}, 0.8173$ ). The variable "age" did not achieve significance (partial f-test, $p=0.97$ ) to increase the power. In this multivariable model, we examined the effect of a discrete PT on GOS score by controlling for GCS score, midline shift, and gunshot wound type. Again, we showed that bitemporal missile tracks (t-statistic $=-2.283, \mathrm{p}=$ 0.023 ) as well as tracks traversing the frontal lobes and entering the contralateral parietal lobe (t-statistic $=-2.311$, $\mathrm{p}=0.025)$ had a significant regression relationship predicting worse outcomes by decreasing the GOS score approximately 1 point per each factor present. In the same model, the GCS score at presentation (t-statistic $=11.994$, $\mathrm{p}<0.001)$ and perforating (t-statistic $=-2.454, \mathrm{p}=0.014$ ), and penetrating (t-statistic $=-0.413, \mathrm{p}=0.044$ ) gunshot wound types were also associated with worse outcomes after adjusting for the remaining explanatory variables. In contrast, midline shift $(\mathrm{t}$-statistic $=0.014, \mathrm{p}=0.988)$ had no significant regression relationship with GOS score.

In another regression model (multiple $\mathrm{R}^{2}, 0.8223$; adjusted $\mathrm{R}^{2}, 0.7817$; predicted $\mathrm{R}^{2}, 0.6542$ ), we included the same previously used variables but removed GCS score from the explanatory variables and calculated the same statistically significant regression relationships as before; however, at that point, the PT that favored survival in the bivariate analysis had become significant: fronto-frontal $(\mathrm{t}$-statistic $=2.816, \mathrm{p}=0.004)$, parieto-parietal $(\mathrm{t}$-statistic $=$ $2.325, \mathrm{p}=0.021$ ), and temporo-temporal (t-statistic $=3.071$, $\mathrm{p}=0.002$ ). When we further attempted to stratify the population by GCS score, we found a strong association of the fronto-frontal and parieto-parietal PTs in subjects with a GCS score $<8$, but no association in subjects with a GCS score $\geq 8$. Furthermore, the stratum-specific estimates differed from one another significantly (chi-square test, $\mathrm{p}=$ 0.002). It appears that there is an effect modification by GCS score in the association between these two PTs and patient survival. The adjusted death ORs for the stratified subjects in the group with GCS scores $<8$ were calculated as fronto-frontal (0.02 [95\% CI 0.00-0.38], $\mathrm{p}=0.022)$, parieto-parietal (0.15 [95\% CI 0.02-0.97], $\mathrm{p}=0.048)$, and temporo-temporal (0.26 [95\% CI 0.01-13.31], $\mathrm{p}=0.858)$.

\section{Surgical Treatment and Length of Hospital Stay}

Of the 73 patients who required surgical intervention, $30 \%(\mathrm{n}=22)$ had an intracranial pressure monitor and/or external ventricular drain (EVD) placed, and $27 \%(\mathrm{n}=20)$ underwent cranioplasty in a delayed fashion. Craniectomy with or without hematoma evacuation was performed in
TABLE 3. Respective intracranial ballistic trajectories that favor death and relative survival odds ratio for each individual path ( $\mathrm{n}=90 / 179$ )

\begin{tabular}{lccc}
\hline $\begin{array}{c}\text { Anatomic } \\
\text { Bullet } \\
\text { Entry Site }\end{array}$ & $\begin{array}{c}\text { Anatomic Bullet Exit Site } \\
\text { or Lodged Intracranial } \\
\text { Compartment* }\end{array}$ & $\begin{array}{c}\text { No. of } \\
\text { Patients }\end{array}$ & $\begin{array}{c}\text { Survival OR } \\
(95 \% \text { CI) }\end{array}$ \\
\hline Parietal & Contralateral parietal & 12 & $0.06(0.00-1.02)$ \\
\hline Temporal & Contralateral temporal & 13 & $\mathbf{0 . 0 5 ( 0 . 0 0 - 0 . 7 9 )}$ \\
\hline Frontal & Contralateral parietal & 16 & $\mathbf{0 . 0 3 ( 0 . 0 0 - 0 . 5 6 )}$ \\
\hline Frontal & Contralateral temporal & 5 & $0.32(0.02-2.24)$ \\
\hline Frontal & Occipital & 5 & $0.32(0.02-3.51)$ \\
\hline Occipital & Contralateral frontal & 3 & $0.29(0.02-6.92)$ \\
\hline Occipital & Contralateral temporal & 2 & $0.55(0.02-13.71)$ \\
\hline Occipital & Frontal & 4 & $0.23(0.01-4.56)$ \\
\hline Occipital & Suboccipital & 2 & $0.55(0.02-13.71)$ \\
\hline Parietal & Contralateral frontal & 3 & $0.29(0.02-6.92)$ \\
\hline Parietal & Contralateral temporal & 7 & $0.10(0.01-1.85)$ \\
\hline Parietal & Occipital & 2 & $0.55(0.02-13.71)$ \\
\hline Parietal & Temporal & 4 & $0.23(0.01-4.56)$ \\
\hline Temporal & Contralateral frontal & 4 & $0.23(0.01-4.56)$ \\
\hline Temporal & Contralateral occipital & 2 & $0.55(0.02-13.71)$ \\
\hline Temporal & Contralateral parietal & 6 & $0.10(0.01-1.85)$ \\
\hline
\end{tabular}

Statistically significant trajectories are shown in bold.

* Refers to ipsilateral intracranial compartments unless stated otherwise.

$27 \%(\mathrm{n}=20)$ of the patients. The remaining $15 \%(\mathrm{n}=11)$ of the patients had a combination of EVD and/or intracranial pressure monitoring followed by craniectomy.

The mean length of hospital stay was 7.5 days, with a median stay of 2 days. Overall, 28.4\% $(n=69)$ of the patients died within a few hours of presentation to the hospital, and 9.5\% $(\mathrm{n}=23)$ died on the first day of admission.

\section{Angiographic Findings}

CT angiography was completed in 54 patients and was found to be negative for large-vessel injury in $70.4 \%(\mathrm{n}=$ 38). In the remaining patients, CT angiography findings were positive for superior sagittal sinus injury in $7.4 \%(\mathrm{n}=$ 4) of patients, transverse sinus injury in 5.6\% $(\mathrm{n}=3)$, internal carotid artery injury in $7.4 \%(n=4)$, middle cerebral artery injury in $3.7 \%(\mathrm{n}=2)$, anterior cerebral artery injury in $1.9 \%(\mathrm{n}=1)$, ophthalmic artery injury in $1.9 \%(\mathrm{n}=1)$, and external carotid artery injury in $1.9 \%(\mathrm{n}=1)$.

\section{Mortality and Primary Outcomes}

Most (82.7\%) patients in our study had an isolated PBI, but $42(17.3 \%)$ presented with multiple gunshot wounds, defined as an isolated PBI and at least one gunshot wound to the body. The mortality rate was $46 \%(\mathrm{n}=112)$ (Table 4). All deaths were directly attributable to PBI, given that we excluded all trauma patients with other causes of death, as we described in Methods. Only 40 (16.5\%) patients were identified as organ donors in our study. Two $(0.8 \%)$ patients remained in a persistent vegetative state at the 3-month follow-up, with a GOS score of 2 . Twenty-one (8.6\%) patients had severe disability and were dependent on others 
TABLE 4. Correlation of GCS score at presentation and GOS at discharge $(n=243)$

\begin{tabular}{cccc}
\hline & \multicolumn{3}{c}{ No. of Patients (\%) } \\
\cline { 2 - 4 } GOS Score & GCS 3-7 & GCS 8-12 & GCS 13-15 \\
\hline 1 & $106(43.6)$ & $6(2.5)$ \\
\hline 2 & $2(0.8)$ & $0(0)$ & \\
\hline 3 & $14(5.8)$ & $6(2.5)$ & $1(0.4)$ \\
\hline 4 & $9(3.7)$ & $9(3.7)$ & $4(1.6)$ \\
\hline 5 & $2(0.8)$ & $18(7.4)$ & $66(27.2)$ \\
\hline Total & $133(54.7)$ & $39(16)$ & $71(29.2)$ \\
\hline
\end{tabular}

to perform their daily activities. Of the remaining patients, $9 \%(\mathrm{n}=22)$ had GOS scores of 4 at the 3-month follow-up, and $35.4 \%(\mathrm{n}=86)$ had GOS scores of 5 and resumed their normal lifestyles despite minor deficits (Table 4).

Among the 112 deaths, 28\% $(\mathrm{n}=31)$ of the patients died after discontinuation of life-sustaining therapies. In a stratified subgroup analysis by withdrawal of care, the adjusted survival ORs for the subjects in the group that did not have discontinuation of care were calculated as bitemporal PTs (OR of survival 0.08 [95\% CI 0.01-0.89], $\mathrm{p}=$ 0.018 ) as well as frontal-to-contralateral parietal PTs (OR of survival 0.05 [95\% CI 0.00-0.68], p < 0.001). The same tracks were not found to be statistically significant in the group that had discontinuation of care.

\section{Analysis of the Entire Sample}

After incorporating all 245 patients in a second analysis, we failed to identify any additional statistically significant associations between variables, or changes in the already established associations other than the ones we described in the main text (Tables 1-3).

\section{Discussion}

PBIs carry high morbidity and mortality., ${ }^{1,511,15,22}$ Both the incidence and mortality following PBI have gradually increased over the last decade. ${ }^{1,2,6,8}$ The mortality of PBIs has been further correlated to PTs, but the various PTs are ill-defined in the literature. ${ }^{4,14,15}$

\section{Demographics and Patient Outcome}

Most studies have reported increased mortality with advancing age. ${ }^{1,714}$ Like other series, we found that most patients with PBI were in their 2nd and 3rd decades, and older patients had worse outcomes in our bivariate model..$^{1,14,15}$ Interestingly, Levy et al. reported that elderly patients had increased survival rates. ${ }^{27}$ Another two studies failed to find any significant association between age and patient outcomes. ${ }^{2,22}$ We solve this controversy in the literature here by referring the reader to our multiple regression analysis where age repeatedly failed to increase the explanatory power in our model. We now conclude that when age is controlled by other significant factors, it does not correlate with worse outcomes as defined by a lower GOS score or death.

There was no relationship between sex and outcome in our series, similar to what other studies have reported. ${ }^{14}$
While Muehlschlegel et al. reported that female sex was a protective factor in PBI, such an association was not demonstrated in our study. ${ }^{28}$

\section{GCS Score at Presentation and Pupil Reactivity}

The GCS score on admission is the strongest predictor of survival. ${ }^{2,3,5,7,22,28}$ It has been previously identified as a significant predictor of outcome, ${ }^{1-5,7,8,11-15}$ and, in our study, patients who presented with a GCS score between 3 and 7 had the worst overall outcomes (OR 68.05 [95\% CI 26.98-171.62], $\mathrm{p}<0.001$ ) (Table 1). Interestingly, only $20 \%(27 / 133)$ of the patients in this group survived, and $59 \%(16 / 27)$ of the survivors developed severe disabilities or progressed to a permanent vegetative state (Table 4).

Pupil reactivity has been a favorable predictor of survival and good functional outcome. ${ }^{3,5,14}$ In contrast, poor outcomes are correlated with bilateral fixed and dilated pupils. ${ }^{4-7}$ Our findings are in line with those in the literature.

\section{Radiographic Findings}

IVH has been associated with mortality in multiple studies. ${ }^{2,5-7}$ We also identified IVH as an important predictor of death (OR 31.25 [95\% CI 11.87-82.29], p < 0.001). It is interesting to report that only $7 \%(5 / 67)$ of the patients with IVH survived. Space-occupying lesions and midline shift in the CT scan were also associated with worse outcomes.

Traumatic pseudoaneurysms may develop within the first hours following PBI or as a delayed complication, with branches of the middle cerebral artery being most at risk. ${ }^{6,28,29}$ Among our patients who underwent CT angiography, $70.4 \%$ had no large-vessel injury.

\section{Significance of Projectile Ballistics in Patient Outcome}

Wounds resulting from PBI can be classified into four major categories: penetrating (entry wound without exit wound), perforating (through and through), tangential, and careening. ${ }^{6,722,23}$ Penetrating and perforating projectiles are statistically significant in contributing to mortality. $5,6,30$ Most studies also have correlated perforating wounds with a poorer outcome and higher mortality rates when compared with penetrating projectiles. ${ }^{5,6}$ In contrast, Can et al. proposed that because the bullet makes a second hole in perforating wounds, it might deposit less energy than a retained bullet, with a consequent reduction in intracranial injury. ${ }^{17}$ Khan et al. reported no difference in outcomes between penetrating and perforating injuries. ${ }^{22}$ In our series, perforating types of injuries had the strongest association with mortality, but penetrating gunshot wound types were still associated with poorer outcomes. Patients with frontofrontal and parieto-parietal penetrating types of PTs had favorable outcomes, especially in the group with a GCS sore $<8$, while patients with a temporo-temporal penetrating type of PT showed favorable outcomes only in the bivariate analysis. Tangential wounds are projectile injuries that do not penetrate through the skull, and they are often associated with the best prognosis. Careening is an unusual injury pattern in which the projectile penetrates the skull, then travels along the periphery of the cortex with- 
out penetrating brain parenchyma. ${ }^{5,6,23}$ We report tangential and careening PBIs as favorable factors for survival.

The degree of bullet fragmentation is affected by bullet construction. Modern handgun bullets reliably expand because of their design, shape, or composition. ${ }^{23-25}$ Expanding (fragmenting) bullets are designed to inflict significantly more tissue damage than nondeformable bullets, ${ }^{23,24}$ and fragmenting bullets are banned from international warfare, but their use by civilians is legal in the United States. ${ }^{24,25}$ Bullets with full metal jackets often remain in one piece and do not leave a trail of lead fragments along their path. Conversely, expanding or fragmenting bullets tend to deform on impact, leaving a telltale trail of metal fragments (Fig. 2). ${ }^{23,24}$ We found expanding projectile types to have a stronger association with unfavorable outcomes compared with nondeformable bullets. Like Khan et al., ${ }^{22}$ we did not find any association between the side of PT and outcomes.

PT has been shown to affect both morbidity and mortality after PBI, 4,7,14,22 and multilobar PBIs have been universally linked to poor outcomes, along with a greater chance of mortality. ${ }^{2,4,14,15}$ Bihemispheric PTs have been associated with even worse outcomes in all series. . $^{2,4,6,8,14}$ Similarly, $94 \%(77 / 82)$ of our patients with a bihemispheric PT did not survive. Bitemporal PTs and bihemispheric PTs through the frontal lobe with the bullet entering the contralateral parietal lobe were universally fatal.

There are rare reports of patients who recovered after bihemispheric injuries. ${ }^{31}$ Khan et al. even reported that bifrontal missile trajectories may be associated with a better prognosis. ${ }^{22}$ We identified 3 patients with bifrontal PTs who survived the insult. One required a hemicraniectomy, remained in the hospital for 49 days, and had a GOS score of 4 at the 3-month follow-up. The second patient also underwent a hemicraniectomy; he was hospitalized for 23 days and had a GOS score of 5 (Fig. 3). The last patient remained in the hospital for 20 days, underwent a cranioplasty, and had a GOS score of 5 on discharge.

Gressot et al. reported posterior fossa PT as a predictor of mortality. ${ }^{14}$ In our model, we failed to associate posterior fossa paths with poor outcomes, which we attributed to the rarity of those PTs. We identified only 2 patients with an isolated posterior fossa PT $(0.8 \%)$, and both survived. Interestingly, one of these patients had expanding projectile fragments in the cerebellar hemisphere, with no associated damage to the brainstem.

\section{Multiple Simultaneous PBIs}

We identified 2 subjects with multiple simultaneous PBIs (Fig. 4). Such reports are extremely rare; the literature is very scant, and the reports are mainly found in forensic journals. ${ }^{32,33}$ The first patient arrived with a GCS score of 3 . He sustained a combination of penetrating and careening types of PBIs caused by two discrete nondeformable projectiles. The second patient surprisingly survived his injuries and had a GOS score of 4 at discharge. He sustained a combination of penetrating types of PTs from nondeformable projectiles (Fig. 4). The patient remained in the hospital for a total of 23 days and was treated with EVD placement. He required a tracheostomy and gastrostomy tube while an inpatient, but nonetheless, at
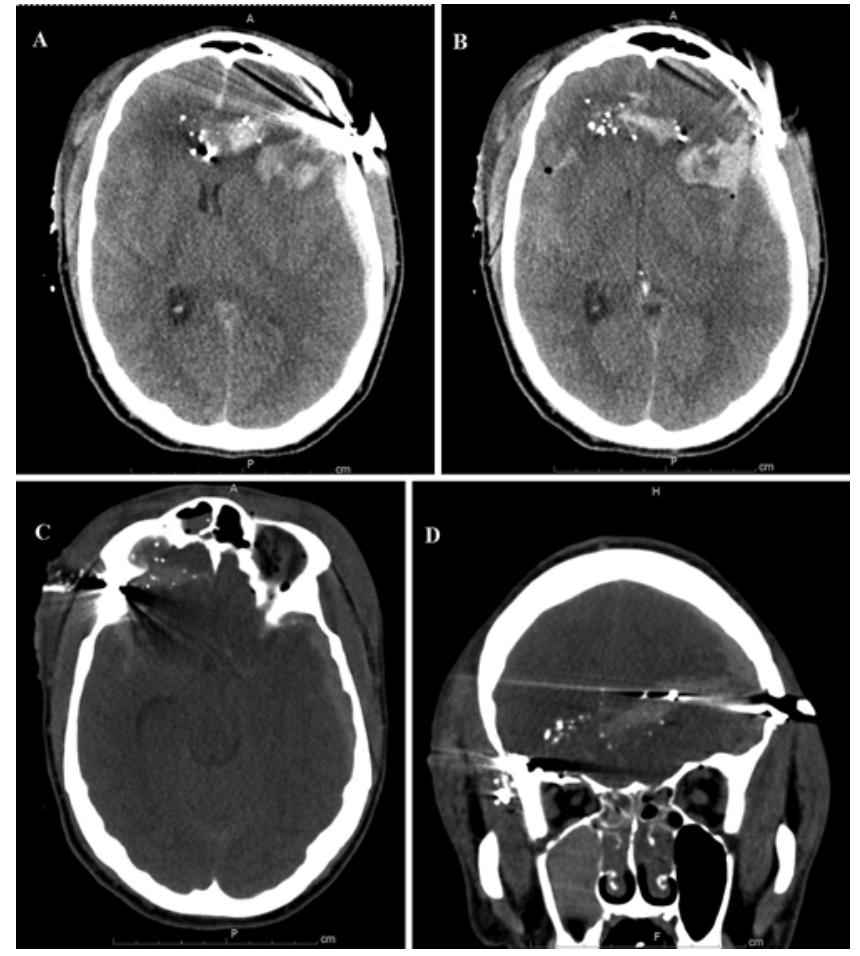

FIG. 3. Example of a 23-year-old survivor following a bifrontal/bihemispheric perforating type of injury caused by a fragmenting type of projectile. A-C: Axial CT scans without contrast demonstrating a left-toright bullet trajectory. There are multiple intracranial missile fragments scattered in bilateral frontal lobes. The patient underwent a left-sided craniectomy and evacuation of the subdural hematoma. He was intact at the 3-month follow-up without neurological deficits. D: Coronal CT scan of the head. The wound is classified as perforating since multiple projectile fragments remain lodged in the scalp by traversing the frontal bone on the right side.

the 3-month follow-up, he could live independently, with moderate disability that included ataxia and minor cognitive deficits. We suggest that patients can potentially survive multiple simultaneous PBIs caused by nondeformable projectiles.

\section{Primary Outcomes After PBI}

Nearly $90 \%$ of the total patients who die after PBI die in the first 72 hours. ${ }^{1-4,6,8}$ In our series, $42 \%$ of the total population, or $91 \%(102 / 112)$ of the patients who died, died of their injuries within the first 48 hours. The long-term outcomes of survivors were generally favorable, with the majority deemed capable of independent living.

Multiple authors have proposed that patients with salvageable injuries necessitate aggressive medical and surgical management. ${ }^{2-5,7,11}$ The Surviving Penetrating Injury to the Brain (SPIN) score is a regression-based risk stratification scale for estimating survival after PBI. ${ }^{27}$ The major controversy against this scale is the exclusion of important radiological factors from the multivariable analysis that, as we proved, remain significant.

There is also controversy concerning surgical management of patients with a GCS score $<8.3,5,7,11$ We showed that patients with penetrating PTs involving the frontal or parietal lobes should be considered for aggressive manage- 

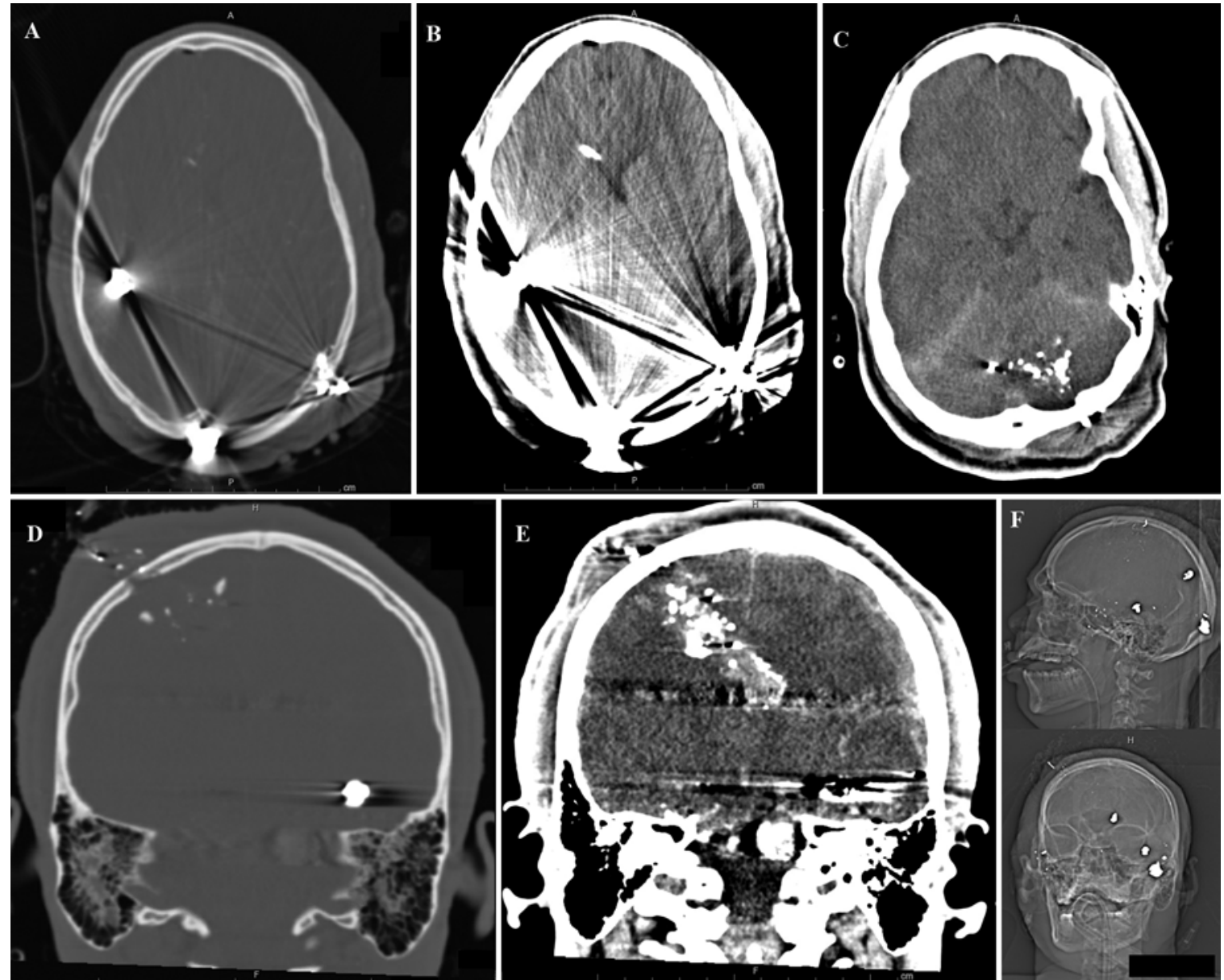

FIG. 4. Multiple simultaneous PBIs related to firearms. A and B: Axial bone (A) and brain (B) window CT scans of the head without contrast. This 29 -year-old male was the sole survivor following multiple simultaneous gunshot wounds. The patient sustained a combination of penetrating and careening types of trauma caused by two discrete nondeformable projectiles with identifiable intracranial paths. One of the projectiles had an entry point to the right occipital lobe and trajectory toward the ipsilateral posterior temporal lobe. C: The second projectile penetrated the skull through the left occipital bone and then traveled along the periphery of the cortex, displacing multiple intracranial bone fragments in the left cerebellar hemisphere. D and E: Coronal bone (D) and brain (E) window CT scans of the head without contrast. This 26-year-old patient had a combination of penetrating types of brain trauma caused by nondeformable projectiles. The first bullet entered the skull through the right parietal bone, and, after crossing the midline, it was found lodged in the left temporal lobe. The second projectile entered the skull through the right greater sphenoid wing, and it was found lodged in the ipsilateral parietal lobe along the interhemispheric fissure. F: Lateral and oblique topogram views of the skull of the same patient.

ment interchangeably with the GCS score at presentation, since there is a survival benefit especially for the group in which the GCS score is $<8$. We recognize as favorable prognostic factors all careening and tangential gunshot wound types, PBIs caused by nondeformable types of projectiles, a higher GCS score at presentation, and a single reactive pupil.

\section{Study Limitations}

The primary limitation of our study is its retrospective design. To eliminate biases, all the information was confirmed by individual chart review. In case of data discrepancy between three CT scan reviewers, our plan was to exclude these patients. As with every retrospective design, our study is subject to type II error and selection bias. To eliminate these, no identified patient with PBI was exclud- ed from the final sample, and the CT scan reviewers were blinded to the patient data. Even though the sample size in this review remains one of the largest in the literature, some trajectories are still underpowered in the study, such as PTs with a suboccipital entry site.

\section{Conclusions}

Trauma patients with penetrating or perforating gunshot wound types associated with bitemporal or frontalto-contralateral parietal PTs should be considered as potential donor candidates. Patients with a favorable PT involving a single frontal or parietal lobe should be considered for early neurosurgical intervention, especially in the circumstances of a low GCS score $(<8)$. Surgeons should not base their decision-making solely on advanced patient age to defer further treatment. Expanding and fragment- 
ing projectiles confer higher mortality rates, while patients with PBIs caused by nondeformable projectiles can survive multiple simultaneous missile trajectories.

\section{References}

1. Skarupa DJ, Khan M, Hsu A, et al. Trends in civilian penetrating brain injury: a review of 26,871 patients. Am J Surg. 2019;218(2):255-260.

2. Kim LH, Quon JL, Cage TA, et al. Mortality prediction and long-term outcomes for civilian cerebral gunshot wounds: a decision-tree algorithm based on a single trauma center. $J$ Clin Neurosci. 2020;75:71-79.

3. Kim TW, Lee JK, Moon KS, et al. Penetrating gunshot injuries to the brain. J Trauma. 2007;62(6):1446-1451.

4. Turco L, Cornell DL, Phillips B. Penetrating bihemispheric traumatic brain injury: a collective review of gunshot wounds to the head. World Neurosurg. 2017;104:653-659.

5. Hofbauer M, Kdolsky R, Figl M, et al. Predictive factors influencing the outcome after gunshot injuries to the head-a retrospective cohort study. J Trauma. 2010;69(4):770-775.

6. Vakil MT, Singh AK. A review of penetrating brain trauma: epidemiology, pathophysiology, imaging assessment, complications, and treatment. Emerg Radiol. 2017;24(3):301-309.

7. Frösen J, Frisk O, Raj R, et al. Outcome and rational management of civilian gunshot injuries to the brain-retrospective analysis of patients treated at the Helsinki University Hospital from 2000 to 2012. Acta Neurochir (Wien). 2019;161(7): 1285-1295.

8. Alvis-Miranda HR, M Rubiano A, Agrawal A, et al. Craniocerebral gunshot injuries: a review of the current literature. Bull Emerg Trauma. 2016;4(2):65-74.

9. Cook A, Osler T, Hosmer D, et al. Gunshot wounds resulting in hospitalization in the United States: 2004-2013. Injury. 2017;48(3):621-627.

10. Fowler KA, Dahlberg LL, Haileyesus T, Annest JL. Firearm injuries in the United States. Prev Med. 2015;79:5-14.

11. Robinson LA, Turco LM, Robinson B, et al. Outcomes in patients with gunshot wounds to the brain. Trauma Surg Acute Care Open. 2019;4(1):e000351.

12. Centers for Disease Control and Prevention. Violence-related firearm deaths among residents of metropolitan areas and cities-United States, 2006-2007. MMWR Morb Mortal Wkly Rep. 2011;60:573-578.

13. Joseph B, Aziz H, Pandit V, et al. Improving survival rates after civilian gunshot wounds to the brain. J Am Coll Surg. 2014;218(1):58-65.

14. Gressot LV, Chamoun RB, Patel AJ, et al. Predictors of outcome in civilians with gunshot wounds to the head upon presentation. J Neurosurg. 2014;121(3):645-652.

15. Darwazeh R, Darwazeh M, Sbeih I, et al. Traumatic brain injury caused by missile wounds in the north of Palestine: a single institution's experience with 520 consecutive civilian patients. World Neurosurg. 2018;116:e329-e339.

16. Tartaglione T, Filograna L, Roiati S, et al. Importance of 3D-CT imaging in single-bullet cranioencephalic gunshot wounds. Radiol Med (Torino). 2012;117(3):461-470.

17. Can Ç, Bolatkale M, Sarıhan A, et al. The effect of brain tomography findings on mortality in sniper shot head injuries. $J$ R Army Med Corps. 2017;163(3):211-214.

18. Puentes K, Taveira F, Madureira AJ, et al. Three-dimensional reconstitution of bullet trajectory in gunshot wounds: a case report. J Forensic Leg Med. 2009;16(7):407-410.

19. Oehmichen M, Gehl HB, Meissner C, et al. Forensic pathological aspects of postmortem imaging of gunshot injury to the head: documentation and biometric data. Acta Neuropathol. 2003;105(6):570-580.

20. Nowinski WL, Thaung TSL, Chua BC, et al. Three-dimensional stereotactic atlas of the adult human skull correlated with the brain, cranial nerves, and intracranial vasculature. $J$ Neurosci Methods. 2015;246:65-74.

21. Nowinski WL, Thaung TSL. A 3D stereotactic atlas of the adult human skull base. Brain Inform. 2018;5(2):1.

22. Khan MB, Kumar R, Irfan FB, et al. Civilian craniocerebral gunshot injuries in a developing country: presentation, injury characteristics, prognostic indicators, and complications. World Neurosurg. 2014;82(1-2):14-19.

23. Jandial R, Reichwage B, Levy M, et al. Ballistics for the neurosurgeon. Neurosurgery. 2008;62(2):472-480.

24. Hakki L, Smith A, Babin J, et al. Effects of a fragmenting handgun bullet: considerations for trauma care providers. Injury. 2019;50(5):1143-1146.

25. Padrta JC Jr, Barone JE, Reed DM, Wheeler G. Expanding handgun bullets. J Trauma. 1997;43(3):516-520.

26. Dsouza LB, Pathan SA, Bhutta ZA, et al. ABC/2 estimation in intracerebral hemorrhage: a comparison study between emergency radiologists and emergency physicians. Am J Emerg Med. 2019;37(10):1818-1822.

27. Levy ML, Masri LS, Lavine S, Apuzzo ML. Outcome prediction after penetrating craniocerebral injury in a civilian population: aggressive surgical management in patients with admission Glasgow Coma Scale scores of 3, 4, or 5. Neurosurgery. 1994;35(1):77-85.

28. Muehlschlegel S, Ayturk D, Ahlawat A, et al. Predicting survival after acute civilian penetrating brain injuries: the SPIN score. Neurology. 2016;87(21):2244-2253.

29. Bodanapally UK, Saksobhavivat N, Shanmuganathan K, et al. Arterial injuries after penetrating brain injury in civilians: risk factors on admission head computed tomography. $\mathrm{J} \mathrm{Neu-}$ rosurg. 2015;122(1):219-226.

30. Aarabi B. Surgical outcome in 435 patients who sustained missile head wounds during the Iran-Iraq War. Neurosurgery. 1990;27(5):692-695.

31. Hazama A, Ripa V, Kwon CS, et al. Full recovery after a bihemispheric gunshot wound to the head: case report, clinical management, and literature review. World Neurosurg. 2018; 117:309-314.

32. Maghin F, Antonietti A, Farina D, et al. A case of suicide by double gunshot wounds to the head: the ability to act after the first shot. Int J Legal Med. 2019;133(5):1469-1476.

33. Karger B, Brinkmann B. Multiple gunshot suicides: potential for physical activity and medico-legal aspects. Int J Legal Med. 1997;110(4):188-192.

\section{Disclosures}

The authors report no conflict of interest concerning the materials or methods used in this study or the findings specified in this paper.

\section{Author Contributions}

Conception and design: Alexopoulos. Acquisition of data: Alexopoulos, Quadri, Bazai, Formoso Pico, Fraser, Kulkarni. Analysis and interpretation of data: Alexopoulos. Drafting the article: Alexopoulos, Quadri, Khan, Kemp, Coppens, Bucholz. Critically revising the article: Alexopoulos, Mercier. Reviewed submitted version of manuscript: Alexopoulos, Quadri, Khan, Kemp, Coppens, Bucholz, Mercier. Approved the final version of the manuscript on behalf of all authors: Alexopoulos. Statistical analysis: Alexopoulos. Administrative/technical/material support: Alexopoulos, Kemp, Coppens, Bucholz. Study supervision: Alexopoulos, Mercier.

\section{Correspondence}

Georgios Alexopoulos: Saint Louis University Hospital, St. Louis, MO.alexopoulos_george@hotmail.com; georgios.alexopoulos@ health.slu.edu. 